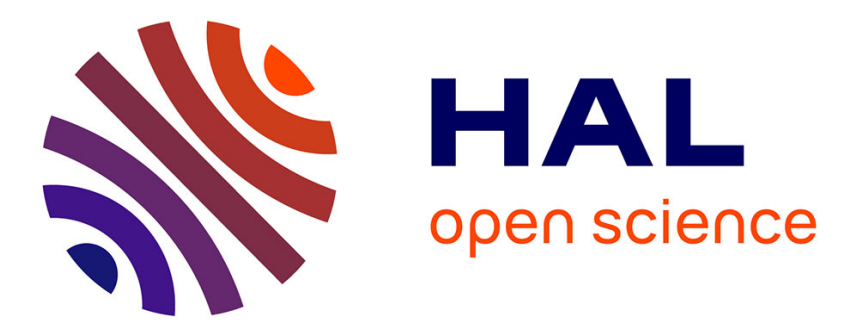

\title{
The size and speed of jet drops are robust to initial perturbations
}

Alexis Berny, Luc Deike, Stéphane Popinet, Thomas Séon

\section{To cite this version:}

Alexis Berny, Luc Deike, Stéphane Popinet, Thomas Séon. The size and speed of jet drops are robust to initial perturbations. Physical Review Fluids, 2022, 7 (1), 10.1103/PhysRevFluids.7.013602 . hal03480945

\section{HAL Id: hal-03480945 https://hal.science/hal-03480945}

Submitted on 15 Dec 2021

HAL is a multi-disciplinary open access archive for the deposit and dissemination of scientific research documents, whether they are published or not. The documents may come from teaching and research institutions in France or abroad, or from public or private research centers.
L'archive ouverte pluridisciplinaire HAL, est destinée au dépôt et à la diffusion de documents scientifiques de niveau recherche, publiés ou non, émanant des établissements d'enseignement et de recherche français ou étrangers, des laboratoires publics ou privés. 


\title{
The size and speed of jet drops are robust to initial perturbations
}

\author{
Alexis Berny ${ }^{1,2}$, Luc Deike ${ }^{2,3}$, Stéphane Popinet ${ }^{1}$, and Thomas Séon ${ }^{1}$ \\ ${ }^{1}$ Sorbonne Université, CNRS, UMR 7190, Institut Jean le Rond d'Alembert, F-75005 Paris, France \\ ${ }^{2}$ Department of Mechanical and Aerospace Engineering, \\ Princeton University, Princeton, New Jersey 08544 USA \\ ${ }^{3}$ High Meadows Environmental Institute, Princeton University, Princeton, New Jersey 08544, USA
}

\begin{abstract}
Bubbles bursting at the ocean surface are an important source of sea-spray aerosol. Indeed, a bubble bursting at the surface of a liquid produces a jet that then breaks up leading to several droplets. Here we simulate the bursting of a single bubble by numerical simulation of the axisymmetric two phases air-water Navier-Stokes equations, in the presence of small initial perturbations in the liquid phase. We describe the impact of this random noise on the size and velocity of the drops for a wide range of control parameters. We then demonstrate how the process of jet drop production is robust to perturbations in the initial conditions, which allows an accurate prediction of the size and speed of jet droplets over the entire parameter space. We propose a mechanism to explain why the existence of some droplets, called "primary droplets" are robust to initial conditions; and discuss why the generation of secondary droplets, much smaller than the primary ones, are sensitive to the same initial conditions. The production of these secondary droplets is sensitive to perturbations in the initial conditions, through a mechanism of pinch-off escape. This can explain previously published experimental observations of bi-modal distributions of jet drops.
\end{abstract}

\section{INTRODUCTION}

Ocean spray, carbonated drinks and many other industrial processes lead to liquid drops produced by bursting bubbles [1, 2]. This very small and rapid event was first observed in a geophysical context with the pioneering studies of Woodcock and Blanchard [3-5]. The importance of bursting bubbles for the exchanges between the ocean and the atmosphere was underlined in subsequent articles from Blanchard et al. [6, 7]. Twenty years later Spiel obtained important statistical results on the size and number of ejected droplets from bursting bubbles in pure water [8] and in salt water [9]. More recently, more experiments have been carried out [10-12] and highspeed cameras now allow to produce very detailed and accurate data on the size and velocity of the first drop as a function of the control parameters [10, 13, 14]. These experimental results have then helped to derive a theoretical framework and scaling laws predicting the drop dynamics [15- -19]. Duchemin et al. carried out the first numerical simulation of an axisymmetric bursting bubble [20]. They revealed that the main parameter controlling the size and the velocity of the first-ejected droplet is the Laplace number (or equivalently the Ohnesorge number) i.e. the ratio between the surface tension and viscous forces. They also identified a resonance phenomenon for a particular value of this dimensionless number where the jet produced the smallest and fastest drops (a few hundreds of meter per second for air in water). Using highly resolved numerical simulations, recent studies have complemented experimental results [12, 21], thus improving our understanding of the bursting bubble process. In particular, Lai et al. [22] proposed a self-similar description of the jetting process history, from cavity collapse to droplet formation. Recently, extending the usual first drop description, we characterised the size and the velocity of all the jet drops produced by bursting bubbles [23, 24]. We demonstrated that they all have to be considered as they can all contribute to the exchanges between the liquid and the gas. Moreover, we observed that, if the description of the first drop, in term of size and velocity, is quite robust, the following droplets seemed to present some variability. This supported an observation first made by Spiel [8, 9] that the drop size distribution might be bi-modal, with two different selected sizes for droplets following the first one.

The primary aim of the present article is to use numerical simulations to statistically estimate the sensitivity to initial conditions of the size and speed of droplets. In a first section we briefly present the numerical methods used in our simulations ( $\mathrm{Sec} \Pi$. We then select three representative cases and describe their drop ejection dynamics (Sec III A). In particular, we show on a jet sequence (Sec IIIB B) how, sometimes, the jet can generate a smaller droplet between two larger drops. We propose a mechanism to explain this sensitivity to the initial conditions. We then statistically explore each case by running a large ensemble of simulations with the same value of the control parameters but with a small perturbation in the initial velocity field. We show that the values of the couple size-speed of each droplet are, most of the time, very robust to perturbations in the initial conditions, making the prediction of size and speed of the jet droplets very reliable. We also highlight the existence of two families of drop populations: large "primary" droplets and smaller "secondary" droplets (Sec IIIC). These two families can explain the bi-modal distributions observed in experiments. Finally, the relative proportion of these two populations is estimated as a function of the control parameters (Sec IIID). 


\section{NUMERICAL SETUP}

We consider two immiscible Newtonian fluids, separated by an interface with constant surface tension. We note $\mu_{i}$ and $\rho_{i}$ respectively the viscosity and the density of fluid $i . \gamma$ is the surface tension coefficient and $g$ stands for the acceleration of gravity. Last, we note $R$ the radius of the bursting bubble. All the parameters are summarized in Figure 1(a). We need four dimensionless numbers to fully describe the problem. We set the viscosity and density ratios to $\mu_{\mathrm{liq}} / \mu_{\mathrm{gaz}}=55$ and $\rho_{\mathrm{liq}} / \rho_{\mathrm{gaz}}=998$, which are close to the values for air and water. For the two remaining numbers, we consider the Bond number Bo, which compares gravitational and capillary forces, and the Laplace number $\mathrm{La}(=1 / \sqrt{\mathrm{Oh}}$, with Oh the Ohnesorge number), which compares capillary forces with viscous forces. They are defined as:

$$
\begin{aligned}
& \mathrm{Bo}=\frac{\rho_{\text {liq }} g R^{2}}{\gamma} \\
& \mathrm{La}=\frac{\rho_{\text {liq }} \gamma R}{\mu_{\text {liq }}^{2}}
\end{aligned}
$$

The initial shape at rest is fully determined by the Bond number. We show an example of an initial shape in Figure 11.a). This equilibrium shape is described by the Young-Laplace equation [13, 25], and we solve it numerically [26]. The shape shown on figure 1 (a) corresponds to a Bond number of 0.01 . Note that the thin liquid film that separates the bubble from the atmosphere is not considered here, which is justified by the very short time of film retraction compared to the characteristic time of bubble bursting. Indeed Ghabache et al. [10] observed that the retraction time of the liquid film lasts for only approximately $100 \mu \mathrm{s}$, whereas it takes one to two milliseconds to produce at least one jet drop.

We perform 2D-axisymmetric simulations with the free software Basilisk library [27]. This software solves the two-phase incompressible Navier-Stokes equations with surface tension. Our numerical method, associated with this initial shape, was already used in a previous work on bubble bursting [23] and are similar to previous work [21, 22, 28,-30]. The adaptive mesh of Basilisk is critical to the success of our simulations as it allows fast solutions for high resolutions equivalent to up to 1638 grid points per bubble diameter.

We initialize the gas and liquid fields with the initial shape of a bubble at rest on a Cartesian mesh with $4^{9}$ grid points which correspond to a resolution of 102 grid points per bubble diameter. In order to study the impact of an initial perturbation, we add an initial noise to the liquid velocity field. For each liquid cell, we impose an initial random velocity $V_{\text {init }}$ using an uniform distribution such that: $-V_{\mathrm{d}} / 100<V_{\text {init }}<V_{\mathrm{d}} / 100$, where $V_{\mathrm{d}}$ is the ejection velocity of the first drop as described in previous studies [21, 23] such that:

$$
V_{\mathrm{d}} \frac{\mu_{\mathrm{liq}}}{\gamma}=k_{v}(1+\alpha \mathrm{Bo})^{-3 / 4} \mathrm{La}^{-3 / 4}\left(\mathrm{La}_{\star}^{-1 / 2}-\mathrm{La}^{-1 / 2}\right)^{-1 / 2}
$$

In this equation, $k_{v}$ and $\alpha$ are fitting parameters equal respectively to 19 and 2.2. $\mathrm{La}_{\star}$ correspond to the critical Laplace number below which no jet drops are produced and is equal to 500. This corresponds to a white noise, with a mean value centered on zero and a noise amplitude of $1 \%$. Other methods to introduce noise or perturbations were tested, either through a small change of the initial Laplace and Bond numbers or modifying the mesh adaptation criteria. All these methods give similar results on the jet drop velocities and sizes. The noise on the velocity field has been selected since its amplitude can be tuned easily. We verified that the statistical results are not sensitive to the amplitude of the perturbation provided it is smaller than a few percent of the first drop velocity. Different methods to produce noise in the simulation are shown in the appendix. In summary, we have verified that the various noise configurations give similar results in terms of the final drop velocities and sizes. In a recent paper focusing on the statistics of jet drops, we have compared the results from these ensembles of simulations with an initial noise set at $1 \%$ of the first drop velocity (see [24]) with the experimental data from Spiel [8]. We have observed a good agreement between the experimental and the numerical jet drop size distribution. Therefore, we consider here an initial random velocity noise with an amplitude of $1 \%$ of the first jet drop velocity.

\section{DROP PRODUCTION RESULTS}

\section{A. Three typical examples of bubble bursting}

Figures 1 (b), (c) and (d) show the evolution of three different simulations, representative of the diversity of drop production dynamics; namely, a Bond number of 0.01 and three Laplace numbers: 2000, 10000 and 100000 (which correspond to air bubbles in water of respectively $0.027 \mathrm{~mm}, 0.14 \mathrm{~mm}$ and $1.4 \mathrm{~mm}$ ). These three Laplace numbers were chosen to cover a range representative of the different typical regimes described for individual 


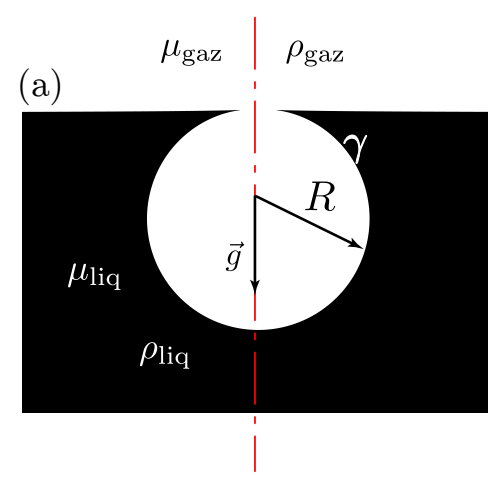

(c) $\mathrm{La}=10000, \mathrm{Bo}=0.01$

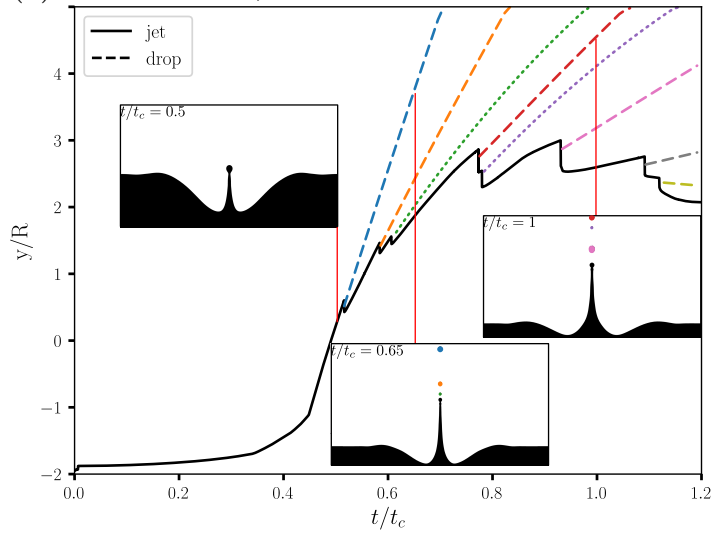

(b) $\mathrm{La}=2000, \mathrm{Bo}=0.01$

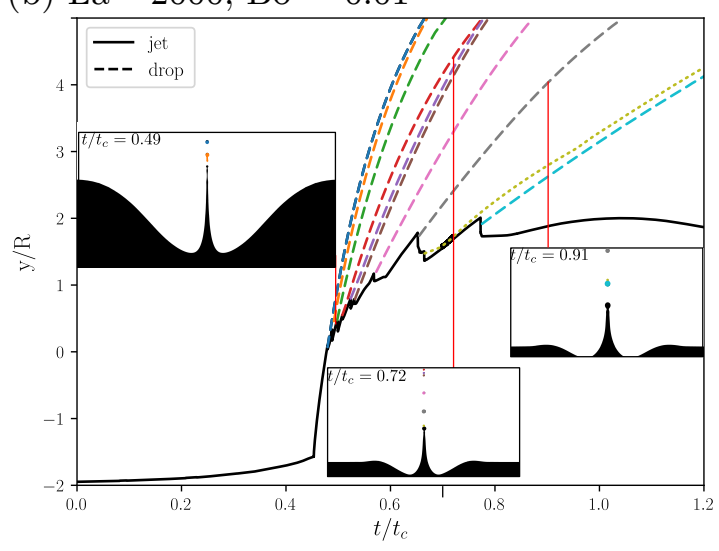

(d) $\mathrm{La}=100000, \mathrm{Bo}=0.01$

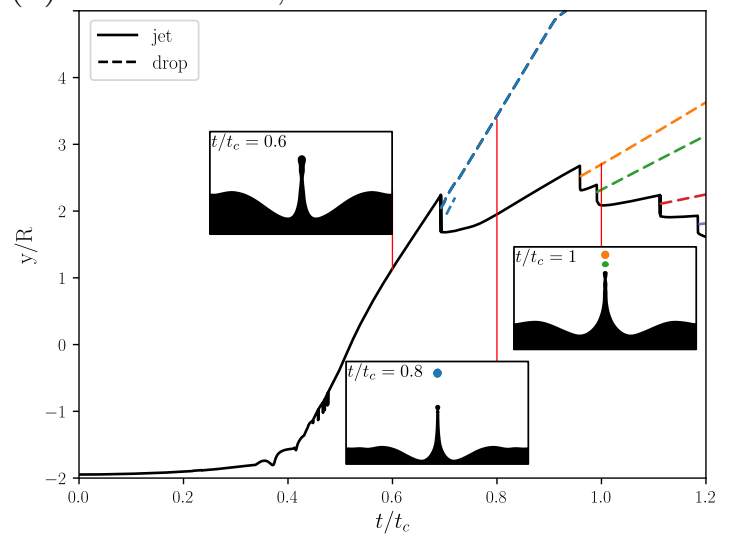

FIG. 1. (a) Initial geometry for a Bond number of 0.01. The initial shape is axisymmetric. We remove the spherical cap of the bubble. We note $\rho_{i}$ the density of phase $i, \mu_{i}$ its viscosity, $\vec{g}$ the acceleration of gravity, $\gamma$ the surface tension between the two phases and $R$ the radius of the bubble.(b)-(d) Evolution of 3 cases. The Bond number is 0.01 for the three cases. The Laplace numbers are: 2000 (b), 10000 (c) and 100000 (d). We plot the evolution of the jet and drop positions along the axis of symmetry. The full line shows the jet position. The dashed lines indicate the positions of the drops. For each case, we display 3 snapshots showing the state of the simulation. The drops are colored with the color used in the graph to show their position.

realizations in terms of number, sizes and velocities of ejected droplets. The Laplace number at 2000 is close to the optimal singularity with the most numerous, fastest and smallest drops and is expected to be the most sensitive to perturbations. The Laplace numbers at 10000 and 100000 correspond to regimes where fewer drops will be produced, respectively 5 to 10 and 1 to 5 , with capillary focusing being less effective. These parameters cover the full range of bubble sizes typically encountered in water, from about $20 \mu \mathrm{m}$ to $2 \mathrm{~mm}$, with associated variations in drop sizes (ranging from 1 to $200 \mu \mathrm{m}$ ), ejection velocities (ranging from 0.7 to $144 \mathrm{~m} / \mathrm{s}$ ), and number of ejected drops (from 1 to 15). As a consequence, these three ensembles allow to probe the role of perturbations on various jet dynamics from bubble bursting and discuss the role of perturbations in the initial conditions.

For each simulation, the position of the jet and drops along the axis of symmetry, normalized by the bubble radius $R$, is presented as a function of time, normalized by the capillary timescale $t_{c}=\sqrt{\rho_{\text {liq }} R^{3} / \gamma}$. The top jet position is represented with a black curve, and the colored dashed lines display the position of the center of mass of the ejected drops. For the three Laplace numbers, three snapshots at different times show the shape of the interface ; the main liquid phase is black and the drops are colored with the same color as the one used for the dashed lines.

As mentioned previously, there is an optimum Laplace number where the jet drops are the smallest and the fastest [12, 20, 21, 23]. For sufficiently small bubbles $(B o \leq 0.01)$ this optimal Laplace number is around 1000. Figure 1 (b) presents a simulation for $\mathrm{La}=2000$, just above this optimal value: we observe six small and fast drops. Their velocities are similar and their sizes also seem to be close, as can be seen on the first two snapshots. We observe the production of a small droplet between two larger drops (snapshot at $t / t_{c}=0.72$ and 0.91 ). Such small droplets can also be found at larger La numbers as can be seen in the snapshots at $t / t_{c}=0.65$ (green drop) and 1 (purple drop) for $\mathrm{La}=10000$. Their trajectory is plotted with dotted lines. The formation of these smaller 
(a) The secondary droplet formation

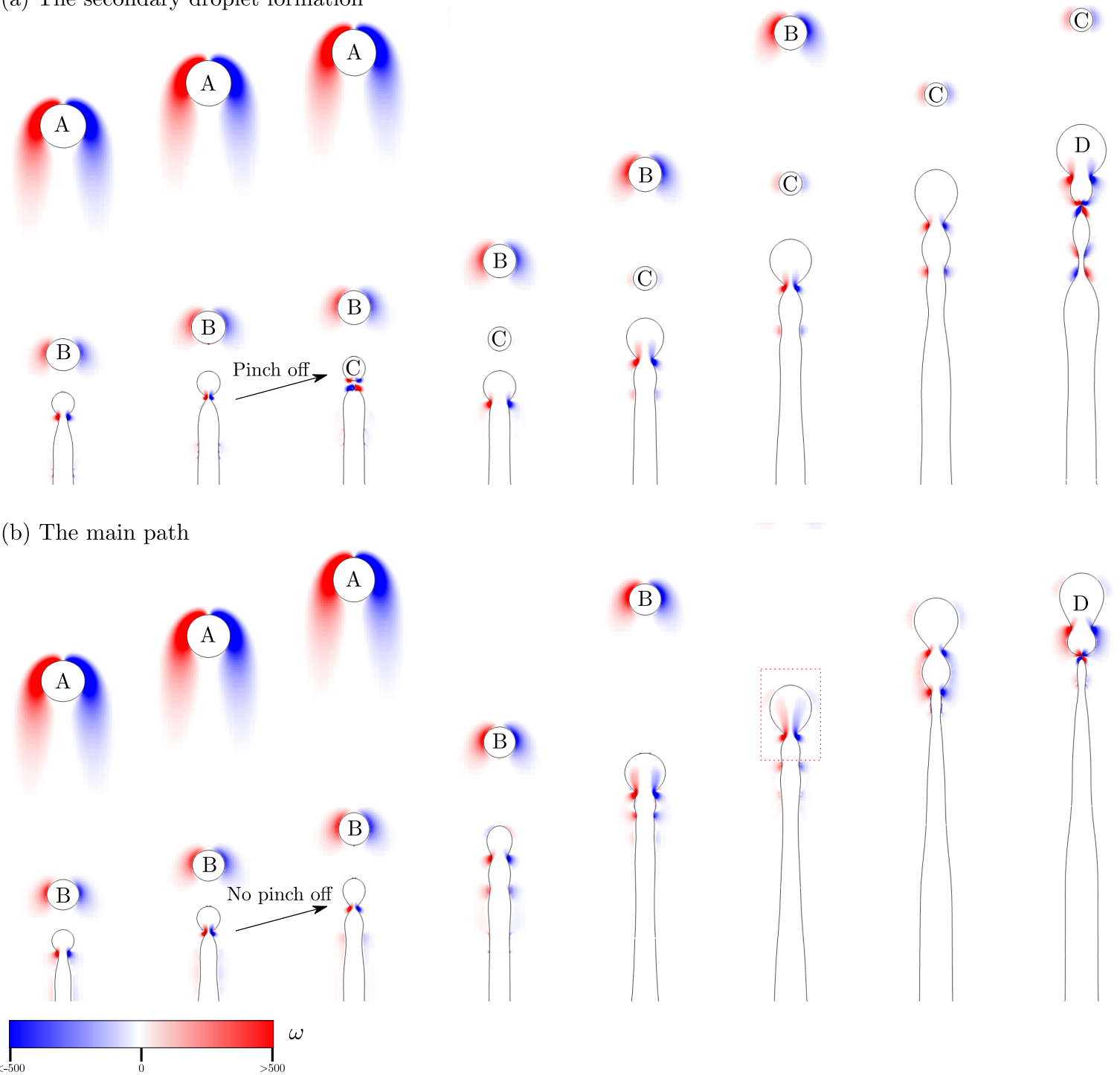

FIG. 2. Two simulations at $\mathrm{La}=10000$ and $\mathrm{Bo}=0.01$ (Fig. 3 (b)). The two simulations only differ in the initial velocity field (randomly perturbed). The top sequence (a) shows the fork mechanism with a first drop in the primary cluster B, then a smaller one in cluster $\mathrm{C}$, before going back to the main path with a drop in cluster $\mathrm{D}$ (see Figure 3 and section $\mathrm{C}$ for a definition of the clusters). The bottom sequence (b) shows the main path, without fork, and the production of a drop in cluster $\mathrm{D}$ following the one in cluster B. The process leading to drop D is the same for the two simulations, despite the formation of drop C in the top simulation.

drops probably explains the bi-modal distribution previously observed in the literature $[8]$ and confirmed in recent studies [24]. One objective of this study is to look at the physical mechanisms that produce the shape of the size distribution and in particular this bi-modality.

For intermediate (c) and high Laplace numbers (d), the number of drops decreases with the La number [23], moreover the initial train of drops no longer exists, the velocity of the drops decreases as they are ejected and the variation of the size is difficult to interpret without a quantitative study. In the following, the production of drops in these cases will be studied quantitatively and, in particular, the sensitivity to perturbations of the initial conditions will be examined.

\section{B. The "fork" phenomenology}

We now focus on the drop production of figure 1( $(\mathrm{c}): \mathrm{La}=10000, \mathrm{Bo}=0.01$, and in particular the formation of the small (green) droplet with a trajectory indicated by a dotted line. Figure 2 (a) presents the details of the evolution of the jet and the formation of the first four drops. The color code of this sequence corresponds to the 
vorticity. Drop A is the first to be ejected, followed by B. The third image shows the jet pinch-off that creates the smaller drop C, corresponding to the dotted line trajectory in Figure 1 (c). The fourth drop D is larger than C.

Figure 2 (b) presents the jet sequence generated with the same control parameters, only the noise in the initial velocity field is different. Consequently, this second sequence is very similar to the first one. In particular, after the detachment of drops A and B, a blob is forming at the top of the jet (second image). However, between the second and the third image, the pinch-off that took place in sequence (a) is avoided and the jet escapes the break up. The exact reason for this difference is not clear, it seems to be linked to the vorticity at the neck of the blob, similarly to the mechanism described by Hoepffner and Pare [31]. In their studies, they observed how a liquid cylinder can or cannot break up, as a function of the cylinder radius. They show that behind the bulb, a few instants after this escape is observed, a vorticity jet can form, similar to the one that appears in the dotted square on the sixth image.

The intriguing result here, is not really the production of the small droplet $\mathrm{C}$, but the fact that the formation of this drop does not change the remaining jet dynamics. Indeed, the rest of the two sequences is very similar: a neck is forming (image 4), its radius decreases, it is about to pinch-off (image 6), it escapes from pinch-off, a new neck is forming below and pinches-off to form drop D.

We call this configuration where the system can form a small droplet or not, depending on the noise in the initial conditions, a "fork". It will be rigorously defined in the next section. In the following this intermediate case (fig. 1. b)) case and the two others presented on the figure 1, are studied by varying the noise in the initial conditions in order to see whether the phenomenon observed here can be generalized or not.

\section{Statistical study of typical examples and bubble bursting robustness}

We consider the three cases highlighted in figure 1: $\mathrm{Bo}=0.01$ and $\mathrm{La}=2000,10000,100000$, and for each case we run, respectively, 118, 115 and 119 simulations with a different random noise in the initial conditions. Figure 3 presents the dimensionless radius of each ejected drop $R_{d} / R$ as a function of its capillary velocity $\mathrm{Ca}=\frac{V_{\mathrm{d}} \mu_{\mathrm{iq}}}{\gamma}$, with $V_{\mathrm{d}}$ the drop velocity. The three graphs stand for the three cases. For each graph, the drops are represented by a colored dot. The color code, given in the insert of figure 3 (a), corresponds to the ejection number of the drops. We observe different regions in the $\mathrm{Ca}-\mathrm{R}_{d} / \mathrm{R}$ phase space where drops from different simulations gather. Most of the regions are encircled in the graphs, with a line when they are well defined and a dotted line when they are more diffuse. We called these regions "clusters" in the rest of the paper. We name them with a letter (A, B...), or a couple of letter (GA, GB...), indicated in the graphs. The existence of these clusters is important as it means that the drop radius and velocity are not selected independently.

Figure 3 (a), presents a bubble bursting event close to the singular case, occurring at $\mathrm{La}=2000$ [20, 21, 23]. In this case, as expected, many drops are produced, up to seventeen. As already observed in figure 1 (a), the first 6-8 drops are ejected very close to one another, they all have a similar small size $\left(\mathrm{R}_{d} \simeq 0.02 \mathrm{R}\right)$, and a high velocity that slowly decreases as the drop number increases [23]. In this region, the drop velocity is significantly sensitive to the initial conditions, as seen on the velocity of the first drop for instance, which ranges approximately from $\mathrm{Ca}=0.9$ to 1.4 . Consequently no clear clusters are formed. We thus define eight areas A, using the drop number in the label: A1, A2, up to A8. The following drops then form six different clusters, with much less noise in the velocity, labeled from B to G. For higher Laplace numbers (fig 3 b) and (c)), except GE and GF in (b) and B in (c), most of the clusters are well defined: the drops are gathered in a small zone of the phase space, indicating that their couple size-speed is selected quite precisely by the system, with a weak sensitivity to initial conditions.

In 2017, Gañán-Calvo built two scaling laws that describe the size and the velocity of the first drops [15]. We recombine these two scaling laws so that an ejection size $R_{d} / R$ corresponds to a given ejection velocity Ca. By adding $20 \%$ error to the ejection size, in order to take into account the dispersion he observed in determining his fitting parameters, we represented his predictions by the grey area in the 3 graphs. The clusters of the first drop (A) show a good agreement with the scaling laws. As these scaling are built for the first drop, the following drops are not expected to fall into the grey area.

The clusters are linked by arrows. These arrows represent the history of the drop production. For example, in figure $3 \mathrm{~b}$ ), the radius and velocity of the 115 first drops that are produced vary such that they form cluster A. After this first drop, every second drop that is formed falls unequivocally in region B. But it is often not as simple and two different scenarios are possible. This is the case after this second drop in cluster B, from which the third drop can be in cluster $\mathrm{C}$ or in cluster $\mathrm{D}$. This bifurcation in the history of drop production is called a "fork". This particular fork is the one detailed in figure 2 , where the only difference between both sequences is the random initial noise. We note that this example is not unique.

It is interesting to note that none of the possible paths cross the grey zone, all the clusters are below. These scaling laws mark a maximum for the droplet size and velocity that the system cannot exceed. Among every paths possible in the graphs, the one that links the biggest drops, the closest to the scaling laws of Gañan-Calvo, is displayed in red and defined as the "main path". The clusters on the main path are called the "primary clusters" and each branch of the main path is called a "main direction". For each fork the percentage of cases following one 

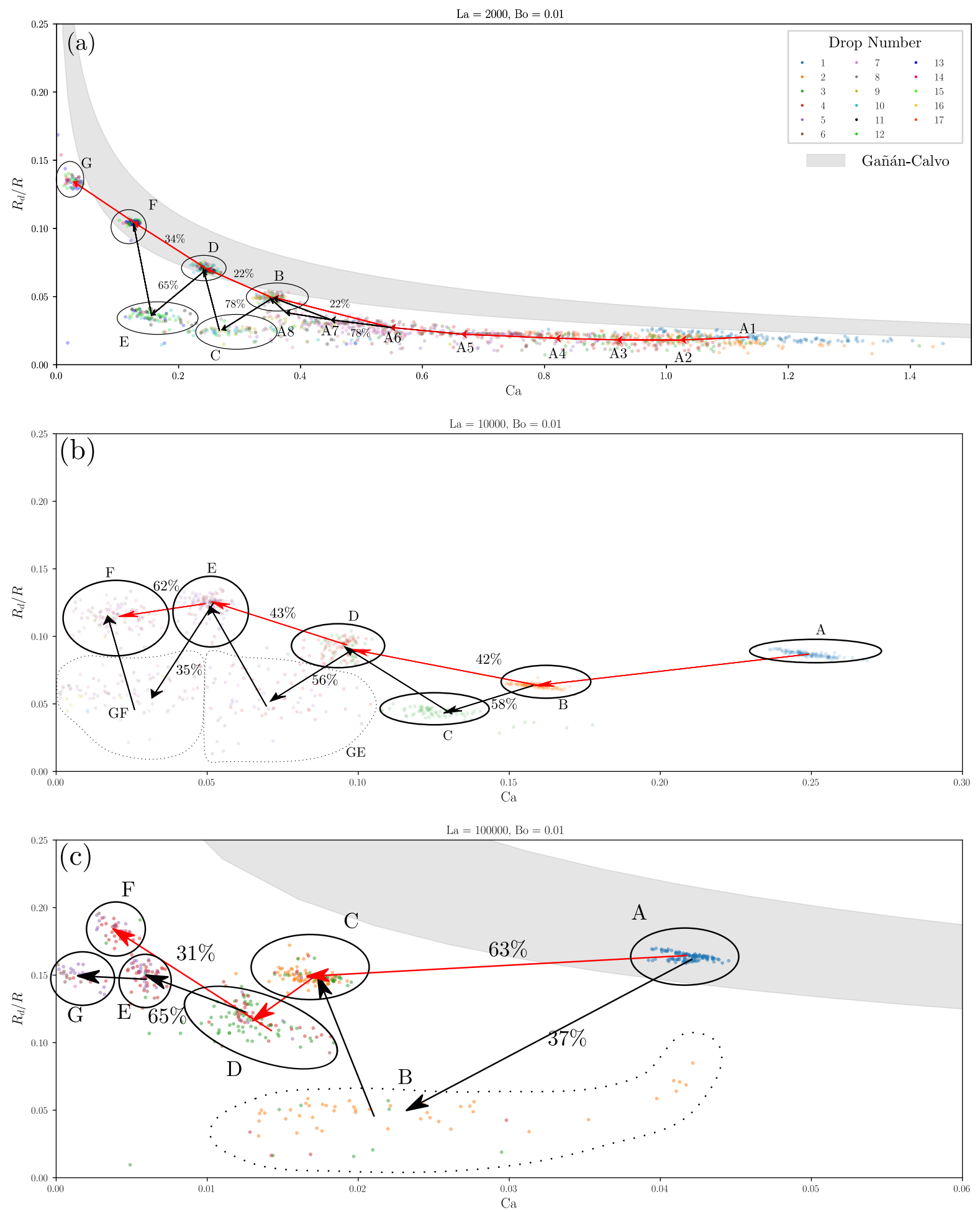

FIG. 3. Dimensionless size of all the drops as a function of their dimensionless velocities. The color code is shown in the insert of figure (a), corresponding to the case $\mathrm{La}=2000$. We identify the different clusters of drops. For each cluster, we indicate the path to the next one. The starting and end points of each arrow are the average speed and sizes of the corresponding clusters. The main path, connecting the "primary clusters", is indicated in red. For each primary cluster, we indicate the percentage of drops following the main path and the percentage of drops following the other paths. The color code shown in insert remains the same for the 3 cases. 
path is written beside the corresponding arrow.

One of the most interesting point of this graph is that every primary clusters will be visited by the drop production process, which is not the case for the secondary clusters that are below the main path. In other words, if at some point the system ejects a small drop belonging to a secondary cluster, the following droplet will be bigger and included in the next primary cluster. For example, when a drop is in the primary cluster B of figure 3 (a) and the following is in C, the next drop will necessarily be in cluster D (a primary cluster). This demonstrates the astonishing robustness of the bursting bubble process. Despite an initial random noise, the drops in the primary clusters will always be produced and their ejection velocity and size can be predicted. These primary clusters form attractors for the drops in the $\mathrm{Ca}-R_{d} / R$ phase space.

To go further in this analysis, the arrows are plotted such that they link the average size and velocity of the drops in the starting and the arrival clusters. Consequently, the arrival points of the different paths in the same primary clusters are not exactly at the same place. However, we observe that they are very close to one another (clusters $\mathrm{B}, \mathrm{D}$ and $\mathrm{F}$ at $\mathrm{La}=2000$, cluster $\mathrm{D}, \mathrm{E}$ and $\mathrm{F}$ at $\mathrm{La}=10000$ and cluster $\mathrm{C}$ for $\mathrm{La}=100000$ ). This means that, for a given bubble bursting event, producing a small droplet included in a secondary cluster does not impact the size and the ejection velocity of the drop in the next primary cluster. This seems to remain true for the entire range of Laplace numbers. This shows again the robustness of the bursting bubble process, in particular the robustness of the main path in the size-speed phase space.

Our only counterexample to the attraction of the main path is in figure 3 (c), at the end of the drop production history. Here, a swerve from the main path (E) does not come back on the main path to end up in a secondary cluster $(\mathrm{G})$. It seems that, in this particular case, when the jet produced a drop in E, it still had enough energy to produce a small drop but not enough to produce the last one in the primary cluster $\mathrm{F}$.

Finally, by looking at the probabilities of escaping the main path, we remark that they seem to decrease as the Laplace number increases. However it is hard to say how the number of drops in secondary clusters evolves. The next section will be devoted to the proportion of secondary droplets and, in particular, to its evolution with the control parameters: Laplace and Bond numbers.

\section{Evolution of the fork probability with the control parameters}

The variability of the droplet production process is characterised mainly by the frequency of formation of drops belonging to the secondary clusters. Consequently, we quantify the probability of formation of these droplets and how this probability evolves with the control parameters. In a set of simulations, $n$ droplets are produced, and among those droplets $n_{\text {main }}$ are in the primary cluster. The proportion of droplets that are not in a primary cluster is then defined as:

$$
q(\text { out })=1-\frac{n_{\text {main }}}{n}
$$

In Figure 4 the evolution of $q$ (out) is plotted with a color scale as a function of the Bond and Laplace numbers. In the whole phase diagram the proportion of drops in a secondary cluster varies between 0 and $45 \%$. As observed in the previous section there is no clear effect of the Laplace number on the sensitivity to the initial condition. In particular the maximum production of secondary droplets does not correspond to the optimal Laplace number but a slightly higher value. As we observed in Figure 3 , this can probably be explained by the impossibility to define clusters for the first ejected drops due to an important variability in their velocity.

The main result we observe in this figure is the strong decrease in secondary drop production when the Bond number increases. Indeed, for Bo $\simeq 0.1$, we observe between 20 and $45 \%$ of drops in the secondary clusters, and for Bo $>0.1$ most of the experiments do not form any secondary drop. In a previous paper we showed that an increase in the Bond number is accompanied by a decrease in drop production (see fig. 5(a) in [23]), here we add that this decrease of drop production is accompanied by a decrease in secondary drop production. When the drops are less numerous the primary clusters are populated first. The results at high Bond number, with few drops being produced are very robust to initial perturbations.

\section{CONCLUSION AND PERSPECTIVES}

Using axisymmetric numerical simulations of the two-phases Navier-Stokes equations, we have studied the sensitivity of the jet drop production by bubble bursting to random perturbations in the initial conditions. The size and speed of the ejected droplets is characterised statistically for a range of control parameters, the Laplace and Bond numbers. Using three particular cases, we highlight that all the drops gather in different but clearly delimited regions in the velocity $(\mathrm{Ca})$ and size $\left(R_{d} / R\right)$ phase diagram. These regions are of two types: primary clusters which contain droplets produced in all cases and secondary clusters which are not systematically visited by the drop production process. The noise in the initial conditions affects droplet production in the secondary 


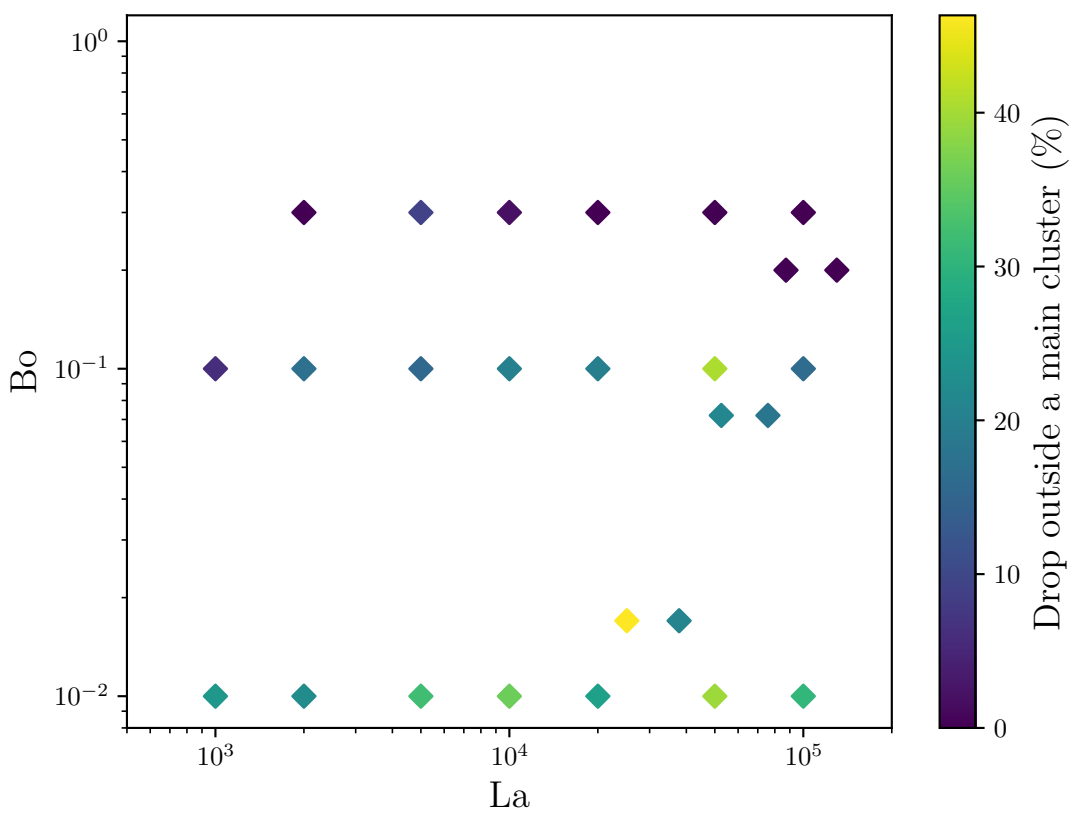

FIG. 4. Probability to observe the production of at least one drop in a secondary cluster, in the La-Bo phase space.

clusters. The parametric study highlights how the probability of droplet production in these secondary clusters is controlled by the Laplace and Bond numbers.

The sensitivity of the secondary droplet production to perturbations in the initial conditions can be discussed in terms of the "pinch-off escape mechanism" highlighted by Hoepffner and Paré [31]. Finally, the existence of these secondary droplets, sensitive to perturbations in the jet dynamics can explain the bi-modal drop size distributions observed in laboratory experiments by Spiel [8, 9] and Ghabache [32].

The robustness of the primary clusters demonstrated in this article, as well as the detailed probabilistic characterisation of secondary droplets, give confidence that practical statistical models of ocean-atmosphere transfers mediated by bursting bubbles can be proposed, as outlined in our recent paper [24] and in [33].

\section{ACKNOWLEDGMENTS}

This work was supported by the National Science Foundation under Grant No. 1849762. to L.D. and the Cooperative Institute for Earth System modeling between Princeton and GFDL NOAA. A.B. was supported by the International Fund to L.D. from Princeton University during part of this study.

\section{APPENDIX: COMPARISON OF DIFFERENT KIND OF NOISE}

We discuss in the present appendix the various types of perturbations or noise introduced in the initial conditions to understand their impact on drop sizes and velocities. All types of perturbations tested led to similar results in the final jet drop size and velocity distributions.

We tested the following two types of noise:

- The first type of perturbation we consider consists in a small change in one of the initial input parameter, in terms of La and Bo number. For example, the Bond number is fixed and we consider a random value for the Laplace number, in a range $\pm 5 \%$ around the desired value. For example, if the Laplace number is set at 25100, we randomly select a Laplace number between 23845 and 26355 . A large ensemble of simulations is then performed and lead to an ensemble of the corresponding ( $\mathrm{La}, \mathrm{Bo})$.

- The second type of noise is the one that is used in the present article: a noise on the velocity. We initialize the velocity field with a small random velocity, which is equally distributed and centered on zero, to ensure that the mean value of the injected velocity is equal to zero. We do that on the original Cartesian mesh, before the adaptation process of the mesh. We set the maximal absolute value of this initial injected velocity at only a few percents of the velocity of the first drop $V_{d_{1}}$. This ejection velocity $V_{d_{1}}$ is computed according to the 

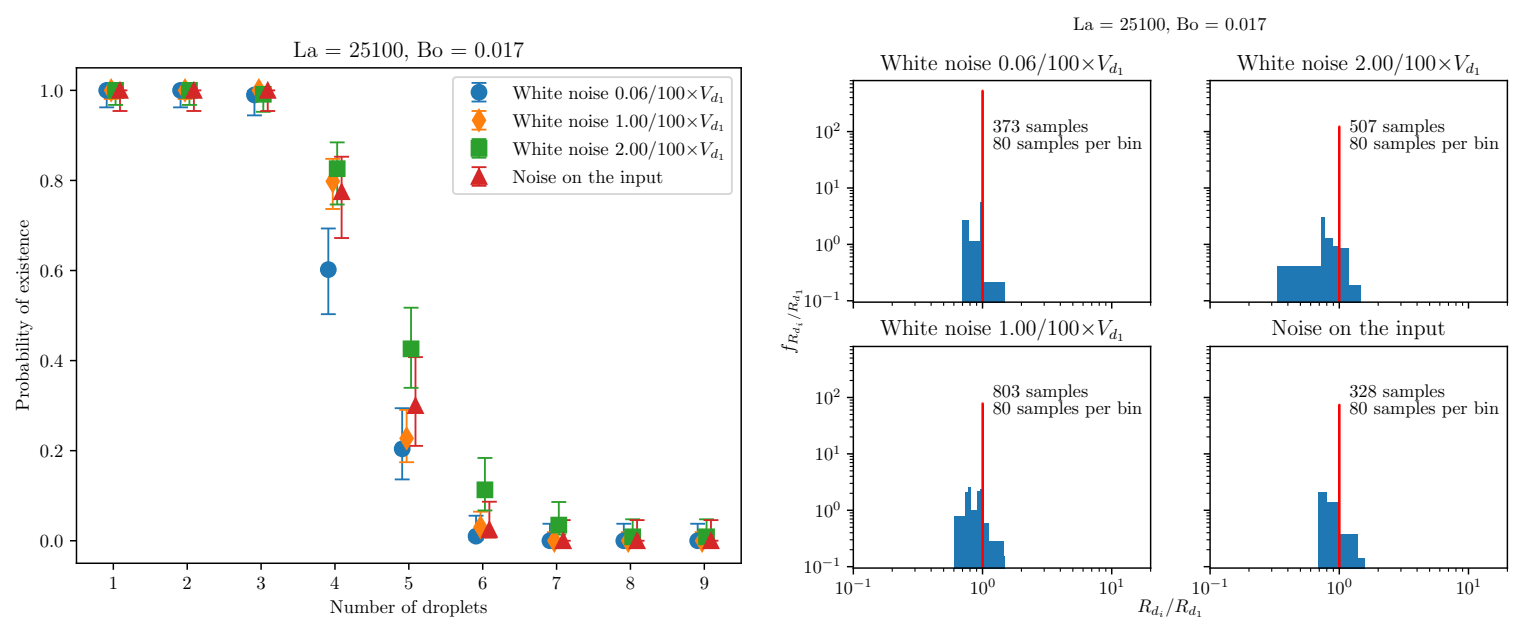

FIG. 5. Left: comparison of the number of ejected droplets for 4 different noises. Three configurations correspond to an initial velocity perturbation with maximum amplitude of $0.06,1$ and $2 \%$ of the velocity of the first drop. The fourth ensemble corresponds to small variations of the Laplace number. We plot for each set of simulations the probability to observe the $i^{\text {th }}$ drop. The error bar correspond to the confidence interval according to a binomial distribution. All the ensembles give similar results. Right: comparison of the size of the ejected droplets with the size of the first one for 4 different perturbations. We plot the probability density function $f_{R_{d} / R_{d_{1}}}$, with on each bin, 80 samples. Again, the simulations sets give similar results, with similar spread and mean values.

scaling of Gañán-Calvo with the correction of Deike et al. equation 5 [21]. We tested different amplitudes for the noise : $0.06 \%, 1 \%$ and $2 \%$ of the first drop velocity $V_{d_{1}}$. This is done on the two dimensions of the 2D-axisymmetric simulation

\begin{tabular}{|c|c|c|c|c|}
\hline Noise & $0.06 / 100 \times V_{d_{1}}$ & $1 / 100 \times V_{d_{1}}$ & $2 / 100 \times V_{d_{1}}$ & Change on Laplace number \\
\hline Number of simulations & 104 & 200 & 119 & 79 \\
\hline
\end{tabular}

TABLE I. Number of simulations for different types of perturbation.

The number of simulations for each noise is summarized in table $\mathrm{I}$ and a comparison of all these results is displayed on figure 5 for a simulation at a Laplace number of 25100 and a Bond number of 0.017. On the left panel, we compare the number of ejected droplets for each type of noise. For each ensemble of simulations we plot the probability to observe the $i^{t h}$ drop. We observe that the probability of existence of each drop is approximately the same for the different ensembles of simulations, demonstrating that the various noises in the initial conditions lead to similar variations in the number of ejected droplets.

On the right panel of figure 5, we plot the probability density function of the size of all the drops compared to the size of the first one (namely $f_{R_{d} / R_{d}}$ ) for each ensemble of simulations. The red bar, centered on 1 corresponds to the size of the first drop. All distributions, for the various types of perturbations in the initial conditions, lead to similar distributions.

The highest one (shown in red) corresponds to the radius of the first drop compared with itself, giving this high and very narrow peak. The second one is located around 0.7 . Then the spread of the data is similar among each distribution. The simulation with an initial noise of $2 \%$ of the first drop velocity shows a biggest spread than the other data, but if we look closely, this spread is captured by only one bin. The simulation set with the noise on the input parameters follow the same trend than the other simulation sets: same peak at 1 and 0.7 , and a similar trend than the simulation set with a noise with an amplitude of $0.01 / 100 \times V_{d_{1}}$.

We have shown that the different types of noise give similar results. In a recent paper, we have compared the simulation set with an initial noise set at $1 \%$ (see [24]) of the first drop velocity with an experimental dataset and obtained a good agreement. Therefore, we choose an initial random velocity noise with an amplitude of $1 \%$ of the initial velocity.

[1] Fabrice Veron. Ocean Spray. Annual Review of Fluid Mechanics, 47(1):507-538, January 2015.

[2] Gérard Liger-Belair. How Many Bubbles in Your Glass of Bubbly? The Journal of Physical Chemistry B, 118(11):31563163, March 2014. 
[3] A. H. Woodcock, C. F. Kientzler, A. B. Arons, and D. C. Blanchard. Giant Condensation Nuclei from Bursting Bubbles. Nature, 172(4390):1144-1145, 1953.

[4] D. C. Blanchard. Bursting of Bubbles at an Air-Water Interface. Nature, 173(4413):1048-1048, 1954.

[5] D C Blanchard and A H Woodcock. Bubble Formation and Modification in the Sea and its Meteorological Significance. Tellus, 9(2):145-158, 1957.

[6] D. C Blanchard. The electrification of the atmosphere by particles from bubbles in the sea. Progress In Oceanography, 1:73-112, IN7, 113-202, 1963.

[7] D. C. Blanchard and L. Syzdek. Mechanism for the Water-to-Air Transfer and Concentration of Bacteria. Science, 170(3958):626-628, November 1970.

[8] Donald E. Spiel. The number and size of jet drops produced by air bubbles bursting on a fresh water surface. Journal of Geophysical Research, 99(C5):10289, 1994.

[9] Donald E. Spiel. More on the births of jet drops from bubbles bursting on seawater surfaces. Journal of Geophysical Research: Oceans, 102(C3):5815-5821, March 1997.

[10] Elisabeth Ghabache, Gérard Liger-Belair, Arnaud Antkowiak, and Thomas Séon. Evaporation of droplets in a Champagne wine aerosol. Scientific Reports, 6(1):25148, July 2016

[11] T. Séon and G. Liger-Belair. Effervescence in champagne and sparkling wines: From bubble bursting to droplet evaporation. The European Physical Journal Special Topics, 226(1):117-156, January 2017.

[12] C. Frederik Brasz, Casey T. Bartlett, Peter L. L. Walls, Elena G. Flynn, Yingxian Estella Yu, and James C. Bird. Minimum size for the top jet drop from a bursting bubble. Physical Review Fluids, 3(7), July 2018.

[13] Elisabeth Ghabache, Arnaud Antkowiak, Christophe Josserand, and Thomas Séon. On the physics of fizziness: How bubble bursting controls droplets ejection. Physics of Fluids, 26(12):121701, December 2014.

[14] Elisabeth Ghabache and Thomas Séon. Size of the top jet drop produced by bubble bursting. Physical Review Fluids, 1(5), September 2016.

[15] Alfonso M. Gañán-Calvo. Revision of Bubble Bursting: Universal Scaling Laws of Top Jet Drop Size and Speed. Physical Review Letters, 119(20), November 2017.

[16] Alfonso M. Gañán-Calvo. Scaling laws of top jet drop size and speed from bubble bursting including gravity and inviscid limit. Physical Review Fluids, 3(9), September 2018.

[17] Alfonso M. Ganan-Calvo and Jose M. Lopez-Herrera. Capillary soft singularities and ejection: application to the physics of bubble bursting. arXiv:1911.08844 [physics], November 2019. arXiv: 1911.08844.

[18] J. M. Gordillo and J. Rodríguez-Rodríguez. Capillary waves control the ejection of bubble bursting jets. Journal of Fluid Mechanics, 867:556-571, May 2019.

[19] Francisco J. Blanco-Rodríguez and J. M. Gordillo. On the sea spray aerosol originated from bubble bursting jets. Journal of Fluid Mechanics, 886:R2, March 2020.

[20] Laurent Duchemin, Stéphane Popinet, Christophe Josserand, and Stéphane Zaleski. Jet formation in bubbles bursting at a free surface. Physics of Fluids, 14(9):3000-3008, September 2002.

[21] Luc Deike, Elisabeth Ghabache, Gérard Liger-Belair, Arup K. Das, Stéphane Zaleski, Stéphane Popinet, and Thomas Séon. Dynamics of jets produced by bursting bubbles. Physical Review Fluids, 3(1), January 2018.

[22] Ching-Yao Lai, Jens Eggers, and Luc Deike. Bubble Bursting: Universal Cavity and Jet Profiles. Physical Review Letters, 121(14), October 2018.

[23] Alexis Berny, Luc Deike, Thomas Séon, and Stéphane Popinet. Role of all jet drops in mass transfer from bursting bubbles. Physical Review Fluids, 5(3):033605, March 2020.

[24] Alexis Berny, Stéphane Popinet, Thomas Séon, and Luc Deike. Statistics of jet drop production. Geophysical Research Letters, 48(10), 2021.

[25] H. Lhuissier and E. Villermaux. Bursting bubble aerosols. Journal of Fluid Mechanics, 696:5-44, April 2012.

[26] http://basilisk.fr/sandbox/aberny/bubble/bubbleShape.h.

[27] http://basilisk.fr.

[28] Luc Deike, Stéphane Popinet, and W. Kendall Melville. Capillary effects on wave breaking. Journal of Fluid Mechanics, 769:541-569, April 2015.

[29] Luc Deike, W. Kendall Melville, and Stéphane Popinet. Air entrainment and bubble statistics in breaking waves. Journal of Fluid Mechanics, 801:91-129, August 2016.

[30] Luc Deike, Luc Lenain, and W. Kendall Melville. Air entrainment by breaking waves. Geophysical Research Letters, 44(8):3779-3787, April 2017.

[31] Jérôme Hoepffner and Gounséti Paré. Recoil of a liquid filament: escape from pinch-off through creation of a vortex ring. Journal of Fluid Mechanics, 734:183-197, November 2013.

[32] Elisabeth Ghabache. Surface libre hors équilibre: de l'effondrement de cavité aux jets étirés. PhD thesis, Université Pierre et Marie Curie-Paris VI, 2015.

[33] Luc Deike. Mass transfer at the ocean-atmosphere interface: The role of wave breaking, droplets, and bubbles. Annual Review of Fluid Mechanics, 54, 2022. 